\title{
Building the centriole
}

\section{cef \\ PP2A has an \\ integral role \\ in centriole \\ assembly by \\ regulating \\ SAS-5.}

Phosphatases are known to play many regulatory parts during the cell cycle. Two groups now show for the first time that, in Caenorhabditis elegans, the phosphatase PP2A has a key role in centriole assembly, a process that is crucial for proper cell division.

During centriole assembly in C. elegans, the kinase zygote defective 1 (ZYG-1) localizes early in the nascent centriole and then phosphorylates spindle assembly abnormal 6 (SAS-6). This ensures the maintenance of SAS- 6 at centrioles and/or the recruitment of SAS-5-SAS-6, the latter leading to the formation of the first centriole structural intermediate,

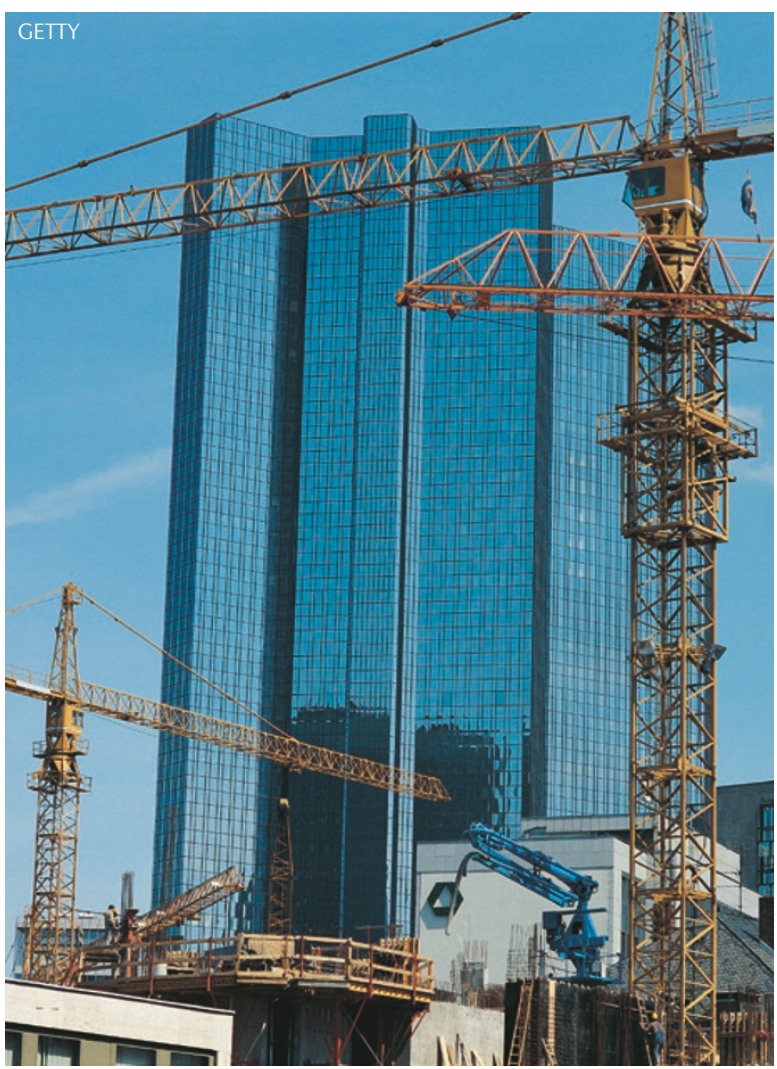

the central tube. As phosphorylation has a key role in the regulation of centriole assembly, the authors postulated that dephosphorylation may also be involved. Indeed, both groups found that RNA interference (RNAi)mediated depletion of LET-92, the PP2A catalytic subunit, gave rise to embryos with mostly monopolar spindles after the second embryonic division, which is indicative of failed centrosome duplication.

PP2A also comprises structural and regulatory subunits, so the authors sought to determine whether these affect centriole assembly. Both groups observed that loss of the structural subunit PAA-1 also led to defects in centriole formation. Furthermore, they identified a partial defect in centriole formation following depletion of the PP2A regulatory subunit SUR-6, although Song et al. attribute this mild effect to incomplete depletion of this protein.

Next, the authors examined how PP2A regulates centriole assembly. Genetic interaction screens showed that let-92 strongly interacts with $z y g-1$ and sas-5 (but only weakly with sas-6), indicating that PP2A may be regulating an early step of centriole assembly. Both groups also identified physical interactions between the proteins: LET-92 co-immunoprecipitated with SAS-5; and, using a yeast two-hybrid assay, Kitagawa et al. found that LET-92 interacts with SAS-5, and that PAA-1 and SUR-6 interact with SAS-6. These findings suggest that the PP2A holoenzyme interacts with SAS-5-SAS-6 early during centriole assembly.

So what is the effect of this interaction? Embryos depleted of LET-92 showed normal localization of ZYG-1 to nascent centrioles, although Kitagawa et al. observed that ZYG-1 levels did not oscillate during the cell cycle, an effect that is also seen with SAS-5 and SAS- 6 depletion. Indeed, both groups found that LET-92 RNAi led to compromised recruitment of SAS-5-SAS-6 to the nascent centriole.

The findings of the two groups point to different, but not mutually exclusive, models of centrosome assembly regulation. Kitagawa et al. observed that, in live embryos, the intensity of SAS-5 tagged with green fluorescent protein decreased from the centriole in the presence of a PP2A inhibitor and that PP2A can dephosphorylate SAS-5 in vitro. Thus, they propose that PP2A-mediated dephosphorylation of SAS-5 is required for its targeting to the centriole. By contrast, Song et al. suggest that PP2A mediates the recruitment of the complex by stabilizing SAS-5, as they found that LET-92 RNAi decreases SAS-5 levels in the embryo.

Regardless of the mechanism, it seems that PP2A has an integral role in centriole assembly by regulating SAS-5. As these proteins have homologues in vertebrates, and Kitagawa et al. show that this may also occur in human cells, the mechanism of centriole assembly regulation by this phosphatase is likely to be conserved.

Rachel David

ORIGINAL RESEARCH PAPERS Kitagawa, D. et al. PP2A phosphatase acts upon SAS- 5 to ensure centriole formation in C. elegans embryos. Dev. Cell 20, 550-562 (2011)|Song, M. H. et al. Protein phosphatase 2A-SUR-6/B55 regulates centriole duplication in C. elegans by controlling the levels of centriole assembly factors. Dev. Cell 20, 563-571 (2011) 